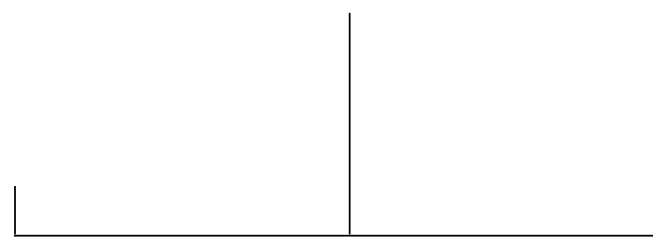

Rev. Latinoam. Psicopat. Fund., São Paulo, v. 15, n. 2, p. 350-358, junho 2012

\title{
Dubois d'Amiens e a sua concepção sistemática da hipocondria e da histeria na França
}

\author{
Sophie Maurissen \\ Mário Eduardo Costa Pereira
}

Frédéric Dubois d'Amiens foi o vencedor do concurso da Sociedade Real de Medicina de Bordeaux em 1830, graças à apresentação da sua tese intitulada "História filosófica da hipocondria e da histeria”. De um ponto de vista existencial, sistemático e por meio de um método histórico-filosófico, ele propõe uma descrição inédita da hipocondria, pois descreve passo a passo a constituição do sujeito afetado, revolucionando assim as concepções médicas e psicopatológicas dessa doença. Apesar das diversas críticas que lhe são apontadas, Dubois d'Amiens reconhece à hipocondria, desde 1830, um sofrimento psíquico e físico específico e vê nela uma forma de pensar, enquanto que Freud a classificará mais tarde como uma neurose atual.

Palavras-chave: Hipocondria, ponto de vista sistemático, Dubois d'Amiens, histeria 
Na primeira metade do século XIX, Frédéric Dubois d'Amiens tornou-se célebre na história da medicina na França por sua crítica da teoria sobre o magnetismo animal. No entanto, uma de suas obras cientificamente mais importantes, embora relativamente desconhecida, trata da questão das relações entre histeria e hipocondria. De fato, esse autor é sobretudo conhecido por seu papel decisivo desempenhado na história do magnetismo, justamente enquanto líder incontestável dos antimagnetistas. Médico formado na Faculdade de Medicina de Paris e membro correspondente da Sociedade Real de Medicina de Bordeaux, Dubois d'Amiens apresenta em 1830 a sua tese intitulada "Histoire Philosophique de l'Hypochondrie et de l'Hystérie" que será vencedora do concurso científico organizado por essa Sociedade (cf. Klein, 2010, p. 132). Ele retoma nesta obra, a classificação nosológica de Boissier de Sauvages segundo ordem, gênero e espécie e impõe uma concepção geral da hipocondria que se insere na evolução das teorias sobre as vesânias desde Pinel (cf. Bercherie, 2004, p. 28).

Dubois d'Amiens nasceu em 1799, na localidade de Amiens, no norte da França. Para se distinguir de seus numerosos homônimos, acrescenta sempre ao seu patronímico o nome da sua cidade da Picardia. Em 1832, depois de ter sido premiado pela Academia Real de Medicina de Bordeaux, Dubois d'Amiens se instala em Paris enquanto secretário perpétuo da Academia de Medicina da França, durante 37 anos. É importante realçar que a tese que este autor apresenta se insere em um contexto histórico no qual a hipocondria é associada exclusivamente à histeria. No início do século XIX, as ideias de Georget sobre esta última dominam as concepções psicopatológicas na França. É nesse contexto que Dubois d'Amiens, as- 
sim como Landouzy, assume uma posição de resistência ao defender as concepções de Pinel e de Villermay, segundo as quais a hipocondria, descrita como uma monomania, seria composta por três períodos distintos. Nesses três, a atenção excessiva que o doente consagra aos seus órgãos daria origem a uma disfunção funcional (primeiro período), neurótica (segundo período) e por fim lesional (terceiro período). A questão da hipocondria e da histeria vai evoluir ao longo do tempo, sobretudo por meio dos diversos concursos abertos pelas grandes Sociedades de Medicina que ilustram o interesse e o mal-estar que continuam a suscitar essas duas doenças pela sua difícil integração na medicina científica. Assim, diversos concursos se seguirão, tais como o de 1830 da Sociedade Real de Medicina de Bordeaux sobre a hipocondria e a histeria, o da Academia de Medicina de 1840 sobre a hipocondria, ou ainda o de 1845 para o Prêmio Civrieux sobre a histeria. Dubois d'Amiens considerava que um novo ensaio era necessário a fim de dissipar a desordem e a confusão instaladas entre a hipocondria e a histeria. Em sua opinião, era necessária a existência de uma crítica filosófica da história dessas duas afeções, da sua prática, bem como era necessária uma apreciação das causas, dos sintomas e dos meios curativos. É com essa convicção que Dubois d'Amiens se consagra; anos antes de apresentar a sua tese, ao seu estudo favorito: as relações entre hipocondria e histeria na história da medicina. Assim, o tema do concurso da Sociedade Real de Medicina de Bordeaux deu-lhe a oportunidade de apresentar em seu estudo todas as noções que suas observações, suas profundas reflexões e sua vasta leitura lhe tinham fornecido. É nesse âmbito que Dubois d'Amiens tentará, com ambição, descrever a causa específica da hipocondria, pois considera que a distinção da sua localização no corpus médico é necessária. Este jovem médico afirma que a região "epigástrica e os hipocôndrios sempre foram mais especialmente designados; mesmo que não se possa de fato encontrar, nessa região, a origem da hipocondria". Segundo ele, os fenômenos característicos desta afecção "vêm da cabeça". Assim, "a originalidade da hipocondria consiste em aliar a um sofrimento da alma feito de uma tristeza e de um receio durável, uma dor física específica situada no hipocôndrio" (Dubois d'Amiens, 1833, p. 11). Afirma ainda que "na hipocondria o princípio intelectual não está afetado", pois o autor considera que na loucura (folie), o fundamento cerebral funcional é distinto do orgânico (cf. Berrios, 2001, p.11).

O capítulo relativo às "Denominações diversas" é fundamental para o leitor situar a posição de Dubois d'Amiens relativamente à hipocondria e à histeria. Numa época em que essas duas estavam intimamente associadas, ele apresenta o seu ambicioso estudo sobre a questão da origem e da natureza da hipocondria e se propõe a distingui-la da histeria. Introduzindo-se no debate acerca da classificação dessas duas patologias, ele apresenta o contexto histórico complexo na qual estas se encontram e situa a sua posição no corpus médico e patológico. 
$\mathrm{Na}$ apresentação que faz do estado dos conhecimentos médicos de seu tempo, que serve como introdução à história da hipocondria e da histeria em sua tese, Dubois d'Amiens desenha rapidamente um quadro histórico da ciência médica, e dá a conhecer sua opinião relativamente às vantagens e aos perigos dos sistemas e dos métodos que os autores tentaram impor antes dele. Recusa seguir unicamente o método experimental, pois acredita que este exclui o raciocínio e tende a reduzir a ciência a listas de fatos que não se encontram interligados. Apresenta-se, então, como um defensor de uma época sistemática, focada essencialmente nas relações existentes entre os diversos dados clínicos. Em sua opinião, os fatos em medicina são recolhidos em demasia de tal maneira que a ciência, essencialmente moral, se encontra sobrecarregada de elementos factuais, mas em falta de uma verdadeira reflexão. Na sua tese, Dubois d'Amiens vai então procurar a ligação sistemática que une os diversos fatos e propõe-se a indicar o caminho a seguir, assim como os diversos obstáculos que se deve evitar para atingir o objetivo da ciência. Sua obra divide-se em seis partes: Considerações preliminares, etiologia, sintomatologia, evolução, natureza essencial e terapêutica. É interessante constatar que em sua tese, Dubois d'Amiens seguiu a estrutura da temática de pesquisa proposta pelo concurso: "Examinar comparativamente as diversas opiniões emitidas sobre a natureza, a origem, a etiologia, a sintomatologia, o prognóstico e a terapêutica da histeria e da hipocondria, assim como realçar a identidade ou a diferença dessas duas doenças" (Falret, 1833, p. 742). No entanto, o autor inverteu a ordem, pois achou judicioso estudar as causas e os sintomas em primeiro lugar, para melhor compreender a natureza essencial e as origens da hipocondria e da histeria. Não podendo aqui fornecer uma análise completa da tese defendida por Dubois d'Amiens, tentaremos apresentar a estrutura da sua obra, assim como as suas principais ideias.

Em sua tese, a parte relativa à etiologia é consagrada mais especialmente à histeria. $\mathrm{O}$ autor afirma que as influências gerais sobre o sistema nervoso fazem parte das causas determinantes dessa patologia. No entanto, não situa a origem da histeria no cérebro, pois este não constitui, segundo ele, a parte mais afetada simpaticamente no ataque histérico. Afirma que os reservatórios do influxo nervoso, agente único dos movimentos, são a medula oblonga (moelle allongée) e a medula espinhal (moelle épinière).

Dubois d'Amiens opõe-se à tese de Foucault sobre as leis do organismo vivo (cf. Falret, 1833, p. 744). Este último defende que a histeria surge apenas quando existe uma lesão simultânea dos órgãos genitais e do encéfalo, enquanto que Dubois d'Amiens afirma que essa lesão suscita violentas convulsões que dão por vezes lugar a um estado de morte aparente, o influxo nervoso tendo ficado momentaneamente esgotado. $\mathrm{O}$ autor trata então de maneira fisiológica a ação dos 
modificadores da economia, distinguindo-se assim da abordagem dos médicos da sua época.

Dubois d'Amiens refere seis variedades de monomania nos sujeitos hipocondríacos, no capítulo relativo à sintomatologia, e classifica-as segundo a sua ordem de frequência. A denominação dessas variedades indica o seu caráter dominante e diferencial: monomania hipocondríaca, pneumocardíaca, encefálica, asténica, nostálgica, hidrofóbica.

Visto que sua tese propõe o estudo sobre as relações entre hipocondria e histeria, o autor apresenta uma tabela comparativa dos sintomas respectivos para tornar mais claras as diferenças entre essas duas afeções. Segundo Dubois d'Amiens, a hipocondria é constituída por três "períodos", enquanto que a histeria é composta por apenas dois "graus". Na sua descrição sintomatológica, ele afirma que a hipocondria surge de maneira lenta e gradual, ao contrário da histeria que em geral aparece sob a forma de ataques súbitos. Apesar dos sintomas dessas duas patologias "afetarem o funcionamento da zona abdominal e dos órgãos da circulação e respiratórios", o autor distingue a sua evolução. Relativamente aos sintomas precursores, ele constata que o sujeito hipocondríaco "centra-se em si" de maneira constante, enquanto que estes são apenas "passageiros" na histeria. Dubois d'Amiens sustenta a hipótese que, no segundo período da hipocondria, diferentes tipos de neuroses tendem a surgir como resultado das afeções do primeiro período, o que não é o caso na histeria. No quadro comparativo apresentado pelo autor, podemos constatar que, de forma geral, o prognóstico da hipocondria é mais grave do que o da histeria. Ele afirma que o sujeito histérico, que se encontra no segundo grau dessa patologia, pode encontrar um estado de "saúde quase completa" enquanto que esse estado de saúde é "quase impossível" para o paciente hipocondríaco que se encontra no terceiro período.

No capítulo sobre a natureza essencial da hipocondria e da histeria, Dubois d'Amiens procura exclusivamente a causa e a origem dessas duas doenças. Depois de ter resumido e discutido as opiniões dos autores sobre a essência das mesmas, desenvolve, de maneira aprofundada, a origem que atribui a essas duas afeções. Começa então pela hipocondria, reunindo as provas que tendem a estabelecer que a maneira de pensar (essência da hipocondria) dos pacientes atingidos por essa patologia não é de todo devida, provocada ou determinada por uma lesão de textura dos órgãos encefálicos, como Georget houvera sugerido. Segundo Dubois d'Amiens, existem tantas modificações mórbidas no cérebro do hipocondríaco, como existem no cérebro de um homem ocupado por qualquer série de ideias com falsas concepções ou que emite falsos julgamentos.

A etiologia, a sintomatologia, a natureza essencial da hipocondria e da histeria são esclarecidas por uma crítica judiciosa do autor na sua tese. Este, em seguida 
aborda então a terapêutica. Nesse capítulo, ele demonstra que os antigos médicos emitiram excelentes observações sobre o tratamento dessas duas patologias. $\mathrm{O}$ mais apropriado na hipocondria e na histeria, segundo Dubois d'Amiens, deve ser baseado na sua natureza essencialmente ideativa, com a adoção de um método racional de tratamento.

A obra a que acabamos de dar uma simples vista de olhos responde, até certo ponto, ao seu título. A história filosófica da hipocondria e da histeria é tratada sob a forma de um debate patológico. Ao premiar a tese de Dubois d'Amiens, a Sociedade Real de Medicina de Bordeaux alertou-o para o fato de que ele isolava demasiado a inteligência do organismo. Ao longo da sua tese, o autor empreendeu um trabalho de construção, pois teve de trilhar e elaborar sua própria teoria a partir do material clínico existente e dos princípios tradicionais, cristalizados no seu tempo. Dubois d'Amiens parece ter realizado essa tarefa complicada, com sucesso.

\section{Referências}

BAILLY, J.-S. Rapport secret présenté au ministre et signé par la Commission précédente. Paris, 1784.

BERChERIE, P. Evolution générale de la notion d'hystérie jusqu'à Charcot - Le sillage de Pinel et ses controverses: Louyer-Villermay, Georget et les Concours. In: BERCHERIE, P. (Org.). Genèse des concepts freudiens: les fondements de la clinique 2. Paris: L'Harmattan, 2004. p. 24-29.

Berrios, G. Hypochondriasis: History of the Concept. In: Starcevic, V.; LipsitT, D. R. Hypochondriasis: Modern Perspectives on an Ancient Malady. Oxford: Oxford University Press, 2001. p. 3-20.

Dubois D'Amiens, F. Histoire philosophique de l'hypochondrie et de l'hystérie. Kessinger Publishing's, 1833. Fac-símile disponível em: <http://gallica.bnf.fr/ark:/ 12148/bpt6k76937p/f1.image>

FALRET, D. M. P. Bibliographie: histoire philosophique de l'hypochondrie et de l'hystérie. Gazette médicale de Paris, Paris, Deuxième série, Tomme 1 1", n. 70, p. 742744, out. 1833.

Guedeney, C.; Weisbrot, C. L'histoire de l'hypocondrie. Monographies de la revue française de psychanalyse, Paris, L'Hypocondrie, p. 29-50, set. 1995.

KLEIN, A. Frédéric Dubois d'Amiens (1790-1873), médecin-philosophe: l'exemple de la question de la Société Royale de Médecine de Bordeaux de 1830. Histoire des sciences médicales, Paris, v. XLV, n. 2, p. 131-145, out. 2010. 
Volich, R.M. A hipocondria: clínica psicanalítica. São Paulo: Casa do Psicólogo, 2002.

\section{Resumos} in France)

(Dubois d'Amiens and his systematic conception of hypochondria and hysteria

In 1830, Frédéric Dubois d'Amiens won a selection process at the Royal Medical Society of Bordeaux with his thesis entitled "Philosophical history of hypochondriasis and hysteria." From an existential and systematic point of view and based on historical-philosophical methodology, the author presents an unpublished description of hypochondriasis by describing, step-by-step, the constitution of the individual suffering from this affection, thus revolutionizing medical and psychopathological conceptions of the disease. Despite severe criticism of his positions, in 1830 Dubois d'Amiens recognized hypochondriasis as a specific psychological and physical disturbance and described it as a way of thinking. Only much later did Freud classify it as an actual neurosis.

Key words: Hypochondriasis, systematic point of view, Dubois d'Amiens, hysteria en France)

(Dubois d'Amiens et sa conception systématique de l'hypocondrie et de l'hystérie

En 1830, Frédéric Dubois d'Amiens remporte le concours de la Société Royale de Médecine de Bordeaux en soutenant son Mémoire intitulé "Histoire Philosophique de l'Hypochondrie et de l'Hystérie". Sous l'angle existentiel et systématique et en s'appuyant sur une méthode historico-philosophique, il propose une description inédite de l'hypocondrie en décrivant pas à pas la constitution de l'individu hypocondriaque, révolutionnant ainsi les conceptions médicales et psychopathologiques de cette affection. Malgré les nombreuses critiques qui lui ont été adressées, Dubois d'Amiens reconnaît à l'hypocondrie dès 1830 une souffrance psychique et physique spécifique et voit en elle une manière de penser, tandis que Freud verra en elle des années plus tard une névrose actuelle.

Mots clés: Hypocondrie, angle systématique, Dubois d'Amiens, hystérie

(Dubois d'Amiens y su concepción sistemática de la hipocondría y de la histeria en Francia)

Frédéric Dubois d'Amiens es el ganador de la competencia de la Real Sociedad de Medicina de Burdeos en 1830, con la presentación de su tesis intitulada "Historia filosófica de la hipocondría y de la histeria”. Desde un punto de vista existencial, sistemático y a través un método histórico-filosófico, propone una descripción inédita 


\section{CLÁSSICOS DA \\ PSICOPATOLOGIA}

de la hipocondría, pues describe paso a paso la constitución del individuo afectado, revolucionando así las concepciones médicas y psicopatológicas de esa enfermedad. A pesar de las varias críticas que le son apuntadas, Dubois d'Amiens reconoce la hipocondría desde 1830 como un sufrimiento psíquico y físico específico y la ve como una forma de pensar, mientras que Freud la clasificará mas tarde como una neurosis actual.

Palabras clave: Hipocondría, punto de vista sistemático, Dubois d'Amiens, histeria

(Dubois d'Amiens y su concepción sistemática de la hipocondría y de la histeria en Francia)

Frédéric Dubois d'Amiens es el ganador de la competencia de la Sociedad Real de Medicina de Burdeos en 1830, con la presentación de su tesis intitulada "Historia filosófica de la hipocondría y de la histeria". A punto de vista existencial, sintomático y a través un método histórico-filosófico, el propone una descripción inaudita de la hipocondría porque describe paso a paso la constitución del individuo afectado revolucionando así las concepciones medicas y psicopatológicas de la enfermedad. A pesar de las varias críticas que le señalan, Dubois d'Amiens reconoce la hipocondría desde 1830 como un sufrimiento psíquico y físico específico y la ve como una forma de pensar, mientras que Freud la clasificará mas tarde como una neurosis actual.

Palabras clave: Hipocondría, punto de vista sistemático, Dubois d'Amiens, histeria

(Dubois d'Amiens und seine systematische Auffassung von Hypochondrie und Hysterie in Frankreich)

Frédéric Dubois d'Amiens war 1830, dank seiner These „Philosophische Geschichte der Hypochondrie und Hysterie“, Sieger des Wettbewerbs der Société Royale de Médecine de Bordeaux. Ausgehend von einer existentiellen, systematischen Sichtweise und anhand einer historisch-philosophischen Methode, schlägt er eine erstmalige Beschreibung von Hypochondrie vor. Er beschreibt Schritt für Schritt das Wesen eines affektierten Menschen und revolutioniert damit die ärztlichen und psychopathologischen Konzeptionen dieser Krankheit. Trotz verschiedener Kritiken, die an ihn herangetragen werden, erkennt Dubois d'Amiens in der Hypochondrie, seit 1830, ein spezifisches psychisches und physisches Leiden und sieht in ihr eine Denkweise, während Freud sie später als eine aktuelle Neurose klassifiziert.

Schlüsselwörter: Hypochondrie, systematische Sichtweise, Dubois d'Amiens, Hysterie

Citação/Citation: Maurissen, S.; Pereira, M.E.C. Dubois d'Amiens e a sua concepção sistemática da hipocondria e da histeria na França. Revista Latinoamericana de Psicopatologia Fundamental, São Paulo, v. 15, n. 2, p. 350-358, jun. 2012. 


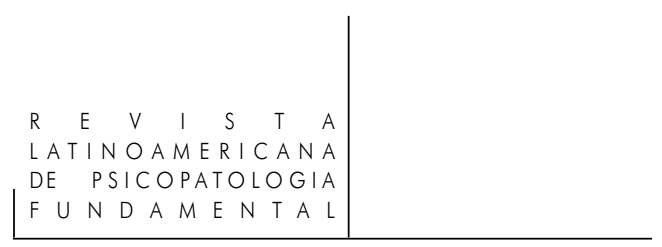

Editor do artigo/Editor: Prof. Dr. Mário Eduardo Costa Pereira

Recebido/Received: 12.2.2012 / 2.12.2012 Aceito/Accepted: 5.4.2012 / 4.5.2012

Copyright: () 2009 Associação Universitária de Pesquisa em Psicopatologia Fundamental/ University Association for Research in Fundamental Psychopathology. Este é um artigo de livre acesso, que permite uso irrestrito, distribuição e reprodução em qualquer meio, desde que o autor e a fonte sejam citados / This is an open-access article, which permits unrestricted use, distribution, and reproduction in any medium, provided the original author and source are credited.

Financiamento/Funding: $\mathrm{O}$ autores declara não ter sido financiado ou apoiado / The authors have no support or funding to report.

Conflito de interesses/Conflict of interest: Os autores declaram que não há conflito de interesses / The authors declare that has no conflict of interest.

\section{Mário Eduardo Costa Pereira}

Psiquiatra; psicanalista; Diretor científico e professor titular de Psicopatologia Clínica do Laboratoire de Psychopathologie Clinique: Langage et Subjectivité da Aix-Marseille Université (Marseille, França); Doutor em Psicopatologia Fundamental e Psicanálise pela Universidade de Paris 7 (Paris, França); Membro da Associação Universitária de Pesquisa em Psicopatologia Fundamental (São Paulo, SP, Brasil); Autor dos livros Pânico e desamparo (São Paulo: Escuta, 1999) e Psicopatologia dos ataques de pânico (São Paulo: Escuta, 2003); Membro do departamento de Psicanálise do Instituto Sedes Sapientiae (São Paulo, SP, Brasil)

Laboratoire de Psychanalyse et Psychopathologie Clinique

Université de Provence

Centre Saint-Charles

Case 37

3, place Victor Hugo

13331 Marseille cedex 3 France

e-mail: mario-eduardo.costa-pereira@univ-provence.fr

\section{Sophie Maurissen}

Aluna do Master em psicologia clínica do Laboratoire de Psychopathologie Clinique: Langage et Subjectivité da Aix-Marseille Université (Marseille, França).

Les Chènevriers

F -05300 Antonaves, France

Rev. Latinoam. Psicopat. Fund., São Paulo, v. 15, n. 2, p. 350-358, junho 2012 